\title{
Molecular hydropathicity index: Application to a series of allosteric modulators of the $M_{2}$ receptors
}

G. Vistoli ${ }^{1, *}$, A. Pedretti ${ }^{1}$, A.M. Villa ${ }^{1}$, L. Villa $^{1}$, U. Holzgrabe $^{2}$ and A. Cambareri ${ }^{2}$

${ }^{I}$ Istituto di Chimica Farmaceutica, Università di Milano, Viale Abruzzi 42, I-20131 Milano, Italy

${ }^{2}$ Pharmazeutisches Institut, Universität Bonn, Kreuzbergweg 26, W-5300 Bonn 1, Germany

The hydropathicity of a series of compounds active as allosteric modulators at the $M_{2}$ muscarinic receptors was calculated and projected on the molecular surface. An analysis of the relationships between conformational and local hydropathicity properties was also performed.

* Author to whom all correspondence should be addressed. E-mail: giulo@indigo.farma.unimi.it

\section{Introduction}

Muscarinic acetylcholine receptors, e.g. $\mathrm{M}_{2}$ receptors, bind allosteric modulators at a site apart from the orthosteric site used by conventional ligands, such as the antagonist $\mathrm{N}$ methylscopolamine (NMS). As a consequence of the binding of the alloster to the receptor, the interaction between the antagonist and the orthosteric binding site is altered [1]. Many of the compounds used to study the 
allosteric modulation belong to the group of neuromuscular blockers, such as alcuronium [2], tubocurarine [3] or gallamine [4] and the alkane bisammonium compound W84. This finding [5] gave the go-ahead of the synthesis of dozens of new compounds, which were derived from W84. All compounds were able to diminish the dissociation rate of the antagonist NMS concentration-dependently without any effect on other receptors. Among others, derivatives of hexamethonium, DUO, WDUO and IWDUO were highly potent [6-10] (see Chart I) and preliminary molecular modelling studies revealed a model of the pharmacophore of the allosteric modulators $[9,11,12]$.

Even though in some cases the lipophilicity of the compounds was characterized as octanol-water partition coefficient, the role of the lipophilicity of the compounds has remained unclear till now. Theoretical calculations according to the classical models of the lipophilicity [13] were not successful due to the fact that the positively charged nitrogens in the molecules and the oxime ether functions, occurring in DUO and WDUO derivatives, are not parameterized. Thus, the purpose of this study was to explore the conformational behaviour of the aforementioned compounds by means of the CHARMM [14] force field and to determine the hydropathicity.

A nearer characterization of the delicate balance that exists between hydrophilic and hydrophobic portions in these derivatives, which contemporarily possess both unitary formal charges and largely hydrophobic moieties, has been attempted by means of an original calculation method, which was developped in our laboratory [15] and since then applied to several series of compounds. This method is able to provide an evaluation of the molecular hydropaticity in a global sense, but also a detailed tridimensional mapping of this property.

\section{Computational methods}

The compounds were built by means of the ChemNote module of Quanta [14] and subjected to a conformational analysis procedure in order to obtain the conformation with the lowest potential energy. The system employed to attribute the force field and the partial charges was CHARMm. The molecules underwent a preliminary minimization with a convergence limit (RMS) of 0.01 , in order to discard the highest energy conformations.

A detailed stochastic conformational analysis was lead with a Monte Carlo type method, which included the random rotation of the dihedral angles which are energetically flexible. This method produced 20 significant conformations among the 20000 generated. A further optimization (RMS = 0.01 ) of these 20 conformations lead to the selection of the one which possessed the lowest potential energy. The best conformation was inserted in a water cluster with a radius of $17 \AA$ (with about 600 solvent molecules) and subjected to a molecular dynamics simulation with a duration of
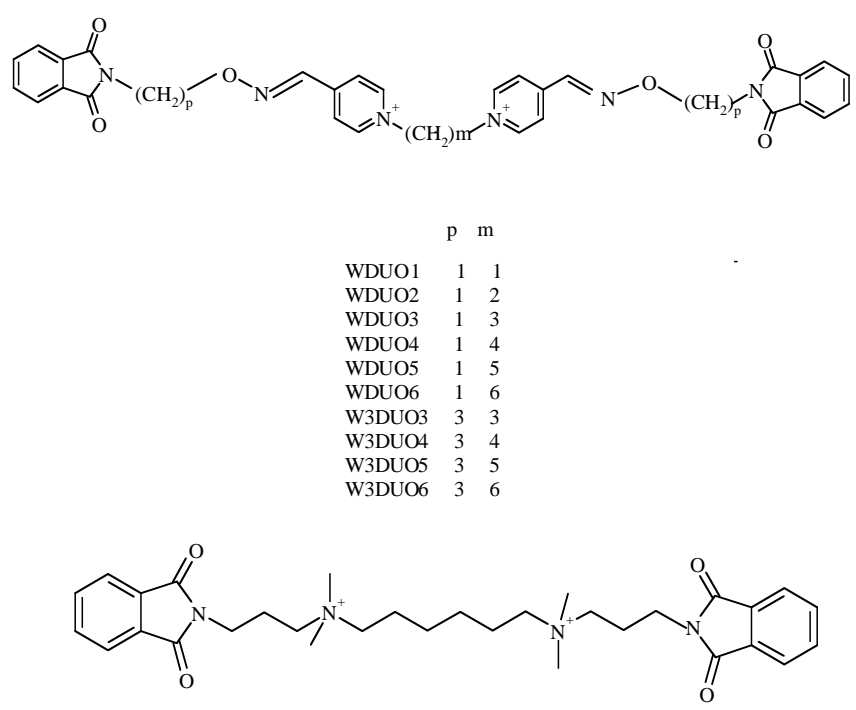

W84
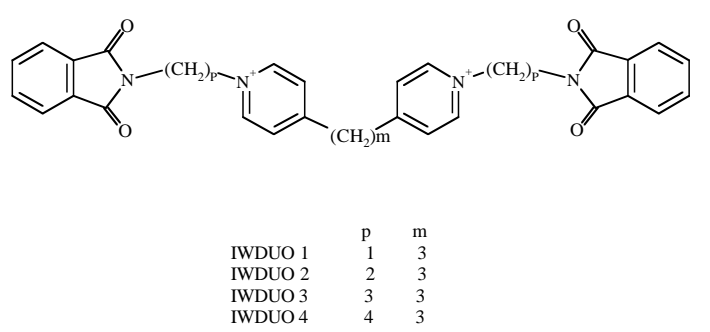

Chart I

$150 \mathrm{ps}$. Again, the lowest energy conformation obtained was minimized again to reach an RMS of 0.01 .

The following step was the calculation of the molecular hydropathicity index ILM according to the methodology developed by Fantucci et al. [15]. In practice the latter is based on the principle that at equilibrium the solvent molecules will be more probably found near the hydrophilic regions of the solute, while they will be repelled by the more hydrophobic moieties, and thus be at a further average distance from the corresponding atoms.

This allows the calculation of a global hydropathicity index (ILM, as said), but this property can also be projected onto a molecular surface, giving rise to a very detailed local hydropathicity mapping. Because of the size of the molecular systems considered in the present work, a further step comprised the correction of the ILM values by exclusion of the contributions of the water molecules which, at the end of the simulation, were found at more than $17 \AA$ from the solute atoms. This is allowed when one takes into account the fact that at such a distance the sum of the contributions of the solvent tends to average out the details in the local hydropathicity profile. 
In the present work, the simulations were performed in an NTE-type canonical ensemble, i.e. maintaining constant the system composition $(N)$, the temperature and the total energy. The temperature window was $300 \pm 50 \mathrm{~K}$. The initial velocities of the atoms were calculated according to the Boltzmann distribution, the time step was 1 fs for a total of 150000 iterations and a frame was saved every $1.5 \mathrm{ps}$ in a trajectory file.

The energy minimizations were performed with a Conjugate Gradient algorithm and included a maximum of 5000 iterations.

For the Monte Carlo analysis, the flexible rotation angles were chosen automatically by the software, the random variation step of the angles was $30^{\circ}$, the temperature was $5000 \mathrm{~K}$ and every 1000 trials a conformation was selected.

\section{Results and discussion}

\section{Conformational analysis}

An analysis of the hydropathicity profile according to the methodology described above is based on a conformational analysis, because of the high degree of conformational freedom of the chain interposed between the two charged moieties in all of the compounds in Chart I.

The main factors affecting the conformation are essentially the mutual electrostatic repulsion among the two charged moieties and the $\pi-\pi$ interaction between the two aromatic portions.

In order to evaluate the conformational behaviour, two geometrical parameters were considered: the distance between the mass centres of the terminal aromatic rings (belonging either to the phthalimido or the dichlorophenyl system) $d \Phi-\Phi$ or else by the distance between the two formally charged nitrogens. The latter value, which has already been shown to be a parameter relevant for activity, is also an indication of the ability of the molecular system to minimize the electrostatic interaction and to reach an energetically stable conformation.

The result of said forces can be observed, e.g. when the behaviour of the WDUO series with respect to the W84 lead compound (see Fig. 1, see page 40). WDUO1 shows a particular conformation due to the particular rigidity of the central, bispyridinic portion. The repulsion between the two aromatic rings forces them at an angle of about $90^{\circ}$ with respect to each other. When the length of the spacer chain between the two charged moieties increases, the WDUO compounds show a tendency to assume an extended disposition until the WDUO3 derivative. A further increase of the chain length leads to more and more folded conformations with a maximum for the derivative WDUO6, where the $\pi-\pi$ interaction between the phthalimido moieties is very strong.
Table I. Conformational Parameters of the M2 allosteric modulators.

\begin{tabular}{lcc}
\hline Compound & $d \Phi-\Phi$ & $d N-N$ \\
\hline WDUO 1 & 19.84 & 2.39 \\
WDUO 2 & 20.85 & 3.17 \\
WDUO 3 & 23.20 & 4.35 \\
WDUO 4 & 20.96 & 4.85 \\
WDUO 5 & 16.14 & 6.37 \\
WDUO 6 & 6.43 & 5.33 \\
IWDUO 1 & 16.68 & 8.90 \\
IWDUO 2 & 19.32 & 9.55 \\
IWDUO 3 & 21.88 & 10.05 \\
IWDUO 4 & 24.74 & 10.15 \\
W 84 & 16.22 & 8.83 \\
W3DUO 3 & 18.34 & 4.821 \\
W3DUO 4 & 20.05 & 6,171 \\
W3DUO 5 & 22.98 & 7.310 \\
W3DUO 6 & 29.04 & 8.914 \\
\hline
\end{tabular}

Also W84, which lacks the pyridinium rings and the oxime systems, shows a relatively folded conformation.

As far as the W3DUO series is concerned, from the table one can see that the W3DUO3 derivative shows an $N-N$ distance which is far higher with respect to the WDUO3 derivative, although the number of carbon atoms between the two pyridine systems is the same. This effect is provided by a higher flexibility of the methylene chain between pyridine and phthalimide moiety, which remarkably differentiates the two series, although the folded conformation is progressively lost with the increase of the chain length.

In the IWDUO series, all derivatives show an extended conformation. The relevant interatomic distances simply increase with the number of carbon atoms included in the spacer chains.

\section{Conformational analysis in solution}

The molecular dynamics simulations performed in water in order to study the hydropathicity profile allowed also to establish that there is no significant modification of the conformational behaviour with respect to the results obtained in vacuo.

Also in this case, the distances between the mass centres of the aromatic systems $(\mathrm{d} \Phi-\Phi)$ and the distances between the two charged nitrogens $(\mathrm{d} N-N)$ were measured for each saved frame in the trajectories.

While the distance between the two formally charged nitrogens keeps oscillating within ranges which are too narrow to allow substantial conformational modifications, the variations of $\mathrm{d} \Phi-\Phi$ present some interesting features. 

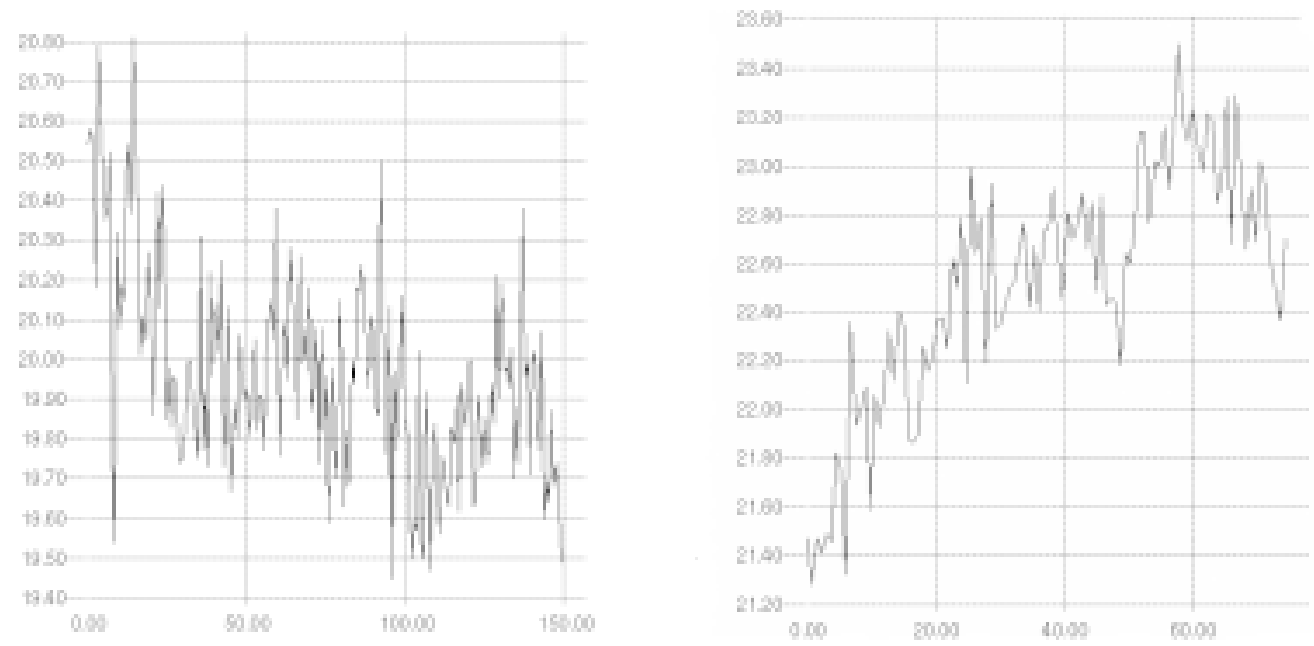

Figure 2. Distances between the mass centers of the aromatic systems $(d(\Phi-\Phi))$ of the homologues series WDUO versus the time of trajectories.
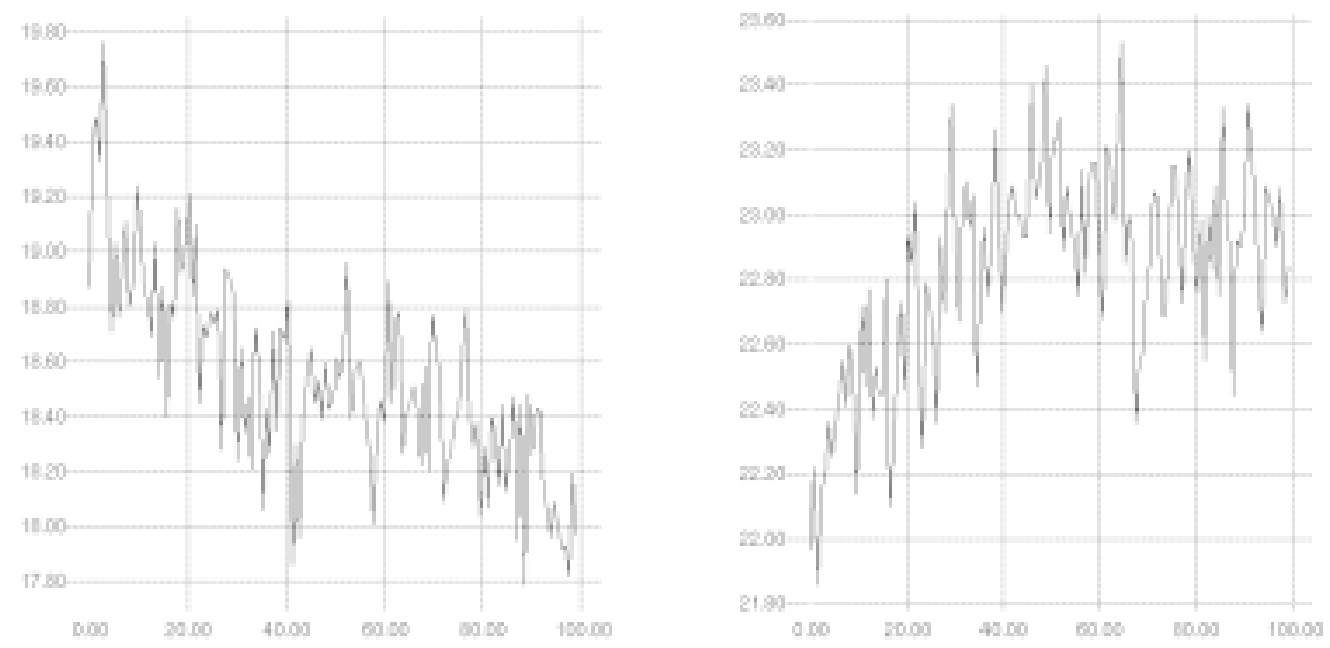

Figure 3. Distances between the mass centers of the aromatic systems $(d(\Phi-\Phi))$ of the homologues series W3DUO versus the time of trajectories.

The results show that in water, the conformational tendencies already shown in vacuo are stressed. An example is the homologous series WDUO. During the simulation of the solvated WDUO1, the aromatic systems of which lie at approximately right angles at the start, the intramolecular distance $\mathrm{d} \Phi-\Phi$ progressively decreases. On the contrary, in the case of the WDUO3 derivative, the starting conformation of which is extended, the same distance is characterized by a progressive increase during the simulation in water. (Fig. 2).
Finally, the WDUO6 compound shows a geometry so folded that $\mathrm{d} \Phi-\Phi$ does not change but within a very narrow range.

Analogously, the derivatives of the W3DUO series show a very similar behaviour. For the W3DUO3 derivatives, which shows a folded, sigmoid conformation at the beginning of the simulation, $\mathrm{d} \Phi-\Phi$ is progressively reduced during the simulation, while the extended W3DUO5 shows the opposite behaviour, i.e. an increase of $\mathrm{d} \Phi-\Phi$ (Fig. 3). 


\section{Hydropathicity profile}

As already pointed out in the methodological section above, the size of the molecules considered brought to the use of a water cluster with a radius of $17 \AA$ for the dynamics simulations. The complexity of the solute-solvent systems thus obtained rendered the calculations particularly demanding from the computational point of view. Moreover, an additional problem was to suitably differentiate the contributions of the water molecules according to their distance with respect to the solute atoms. This was achieved by "normalizing" the surroundings of each solute atom as described above. The most accurate results were obtained if the cutoff beyond which the solvent influence can be neglected were set equal to the radius of the used cluster, in this case $17 \AA$.

In table II one can also observe that, in agreement with the expectations from the conformational analysis, e.g. for the WDUO series, a progressive increase of the $I L M_{\text {cutoff }}$ with the increase of the length of the methylene chain is obtained. For the WDUO6 derivative, which has a remarkably more folded conformation, the surface of the apolar moieties exposed to the solvent is reduced, and, consequently, a lower $I L M_{\text {cutoff }}$ value is calculated.

When these values are compared to the conformational parameters in table I, a correlation can be observed between the $N-N$ distances in table I and the $I L M_{\text {cutoff }}$ values. On the other hand, this is not true for the $\mathrm{d} \Phi-\Phi$ distances of the phthalimidic structures.

If the spatial distribution of the hydropathicity property is considered by the analysis of its projection on the molecular surfaces of the compounds, the influence of the conformational situation appears to be of critical importance. Indeed, for instance in the WDUO series, one can observe a progressive increase in the $N-N$ interatomic distance with the increase of the number of methylene units of the spacer portion, while the distance between the mass centres of the aromatic systems follows a parabolic trend, due to the progressive packing of the two aromatic subsystems.

As expected, the interpyridinic spacer shows a more and more hydrophobic character, while the most hydrophilic portion is located in proximity of the charged moieties (see Fig. 4, see page 40). On the other hand, the folded derivatives show a behaviour that is somehow independent on the local polarity of the groups which build up the molecule: in general, the external, convex part of the molecular surface is less hydrophilic when compared to the concave portion. This is due to the U-shape of the surface, where the two symmetric moieties tend to trap some solvent molecules between them. This situation changes in the case of the WDUO6 derivative, where the spacer chain is long enough to allow the hydrophobic collapse of the molecule and the expulsion of every solvent molecule from the space between the two symmetric portions.

In the W3DUO (Fig. 5, see page 41) and the IWDUO series, the conformations are extended; therefore, the phthalimidic portions result to be more hydropathic due to
Table II. Hydropathicity IL $M_{\text {cutoff }}$ values for the $M_{2}$ allosteric modulators.

\begin{tabular}{lccc}
\hline Compound & $I L M_{\text {cutoff }}$ & Compound & $I_{\text {cutoff }}$ \\
\hline WDUO1 & 3.35 & IWDUO2 & 3.99 \\
WDUO2 & 3.94 & I WDUO3 & 4.29 \\
WDUO3 & 4.04 & I WDUO4 & 4.33 \\
WDUO4 & 4.58 & W84 & 4.55 \\
WDUO5 & 4.77 & W3DUO3 & 4.14 \\
WDUO6 & 4.63 & W3DUO4 & 4.43 \\
I WDUO1 & 3.72 & W3DUO5 & 4.94 \\
\hline
\end{tabular}

the simple fact that the solvent molecules are spatially less far from the outer boundaries of the solute.

Some preliminary results were obtained with an originally developed method [16] which allowed to compare the hydropathicity profile of some of the bisammonic derivatives with alcuronium. The latter is usually considered as a rigid template for allosteric $\mathrm{M}_{2}$ modulators. In figure 6 (see page 41), one can see the superposition of the compounds onto alcuronium. The colour coding corresponds to the degree of similarity of the hydropathicity values (red for maximum, blue for minimum similarity). This representation allows to see that the highest overlap of the hydropathicity property can be found in the regions near the charged moieties, whereas it diverges for all other molecular areas.

\section{Conclusions}

In summary, the results obtained point to a range of qualitatively differences as far as the conformational behaviour, both in vacuo and in solution, and the hydropathicity profile among the bisammonic compounds are considered. This suggests that they may bind in quite different ways to the biological target, although sharing some common substructures. However, no quantitative correlation with the allosteric activity was possible. This is a hint to the fact that the role of the hydropathicity profile is important, but complementary to that of other molecular descriptors in the description of the complex mechanism of allosteric modulation.

\section{References}

1. Holzgrabe, U.; Mohr, K. Drug Discovery Today 1998, 3, 214222.

2. Proska, J.; Tucek, S. Mol. Pharmacol. 1994, 45, 709 - 717 and references cited herein.

3. Waelbroek, M.; Robberecht, P.; De Neef, P.; Christophe, J. J. Recept. Res. 1988, 8, 787 - 808.

4. Stockton, J. M.; Birdsall, N. J. M.; Burgen, A.S.V.; Hulme, E.C. Mol. Pharmacol. 1983, 23, 551 - 557. 
5. Jepsen, K.; Lüllmann, H.; Mohr, K.; Pfeffer, J. Pharmacol. Toxicol. 1988, 63, 163-168.

6. Botero Cid, M. H.; Holzgrabe, U.; Kostenis, E.; Mohr, K.; Tränkle, C. J. Med. Chem., 1994, 37, 1439-1445.

7. Kostenis, E.; Holzgrabe, U.; Mohr, K. Eur. J. Med. Chem. 1994, 29, 947-953.

8. Gasteiger, J.; Holzgrabe, U.; Kostenis, E.; Mohr, K.; Sürig, U.; Wagener, M. Pharmazie 1995, 50, 99-105.

9. Kostenis, E.; Botero Cid, H. M.; Holzgrabe, U.; Mohr, K. Eur. J. Pharmacol. 1996, 314, 385-392.

10. Holzgrabe, U.; Mohr, K.; Staudt, M.; Tränkle, C. Life Sci. 1998, 62, 423-429.

11. Holzgrabe, U.; Wagener, M.; Gasteiger, J. J. Mol. Graph. 1996, 14, 185-193.
12. Holzgrabe, U.; Hopfinger, A. J. J. Chem. Inf. Comp. Sci. 1996, 36, 1018-1024.

13. Leo, A. J. in: Hansch, C.; Sammes, P.G. and Taylor, J.B. Eds., Comprehensive Medicinal Chemistry: "The rational design, mechanistic study \& therapeutic applications of chemical compounds", Vol. 4, Pergamon Press, Oxford, England, 1990, Chapter 18.7; pp 295-319.

14. Quanta, Molecular Simulation Inc., Burlington, MA, USA.

15. Fantucci, P.; Villa, A. M.; Villa, L.; Vistoli, G. Trends in QSAR and Molecular Modelling, Sanz, F. Ed., Prous Barcelona (1995).

16. Vistoli, G. "A new three-dimensional parameter of molecular hydropathicity", Univ. Milan, Ph. D. Thesis (1998). 

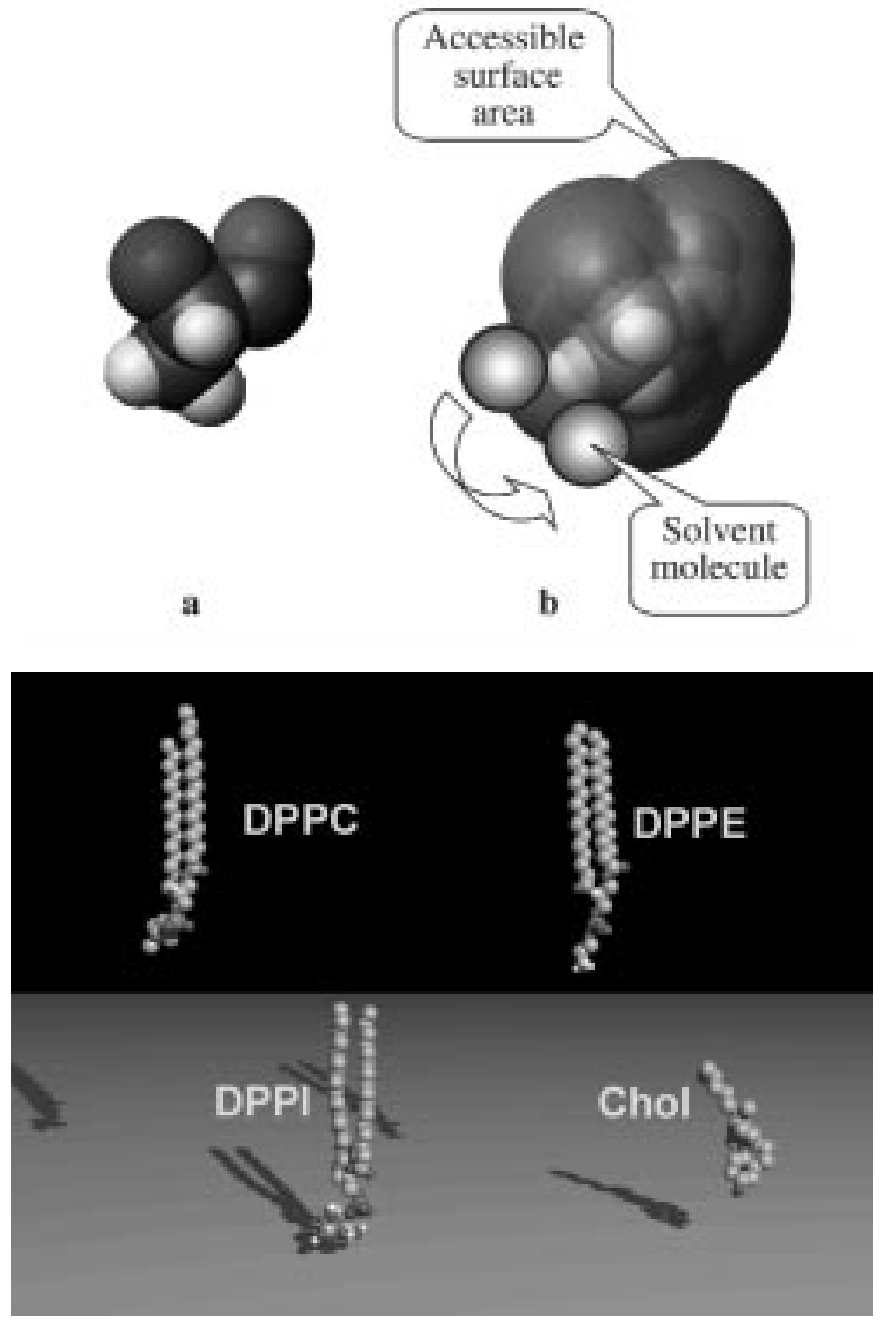

Figure 2. a) Atomic description of alanine. b) Water-accessible surface of alanine is plotted as the surface described by the centre of a sphere representing a solvent molecule of 1.4 Angström radius and rolling on the molecule (see article Van Eyck et al.).

Figure 3. a) Representation in real volume (CPK) of four lipids existing in natural membranes. DPPC: dipalmytoyl phosphatidyl choline. DPPE: dipalmytoyl ethanol amine. DPPI: dipalmytoyl phosphatidyl inositol. Chol: cholesterol. b) Visualisation of isopotential surfaces of molecular hydrophobicity (MHP) around the four previous lipids, in the same orientation as in part a. The orange surfaces are the hydrophobic isopotential area, the acyl chains; the green surfaces are the hydrophilic ones, the polar heads (see article Van Eyck et al.).
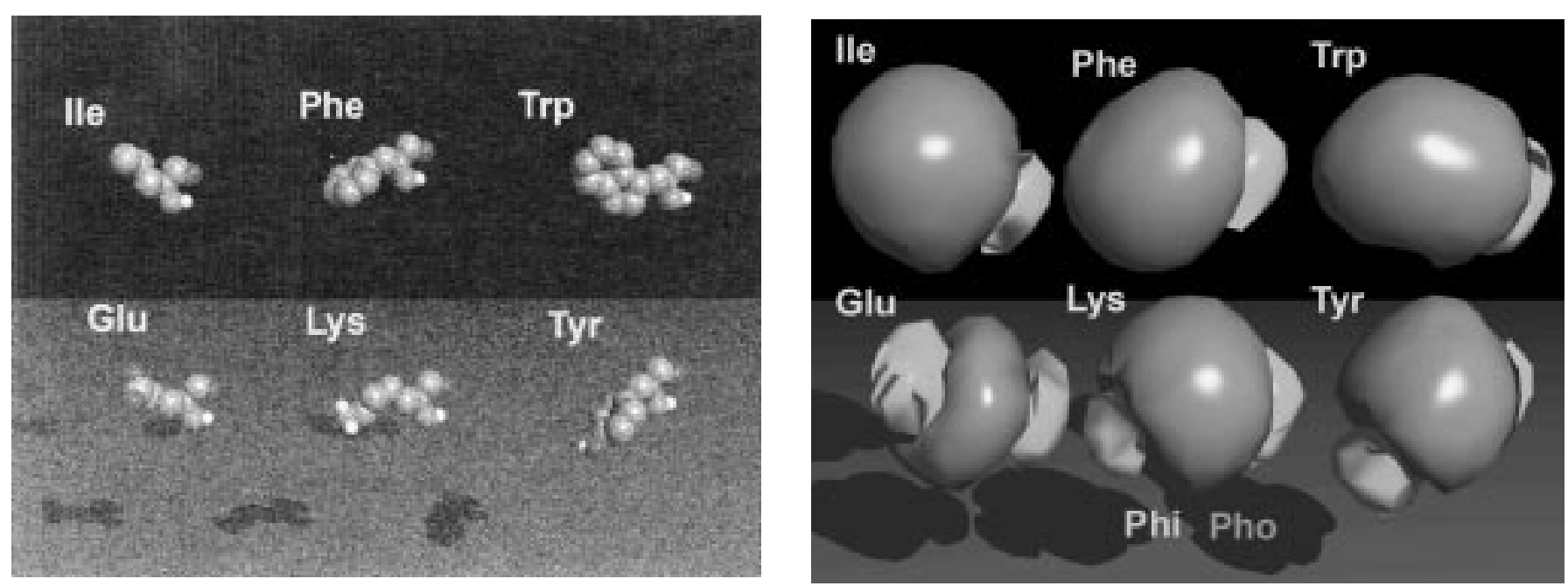

Figure 4. a) Representation in real volume (CPK) of 6 amino acids, 3 hydrophobic (lle, Phe and Trp) and 3 polar (Glu, Lys and Tyr). b) Visualisation of isopotential surfaces of molecular hydrophobicity (MHP) around the 6 amino acids in the same orientation as in part a. The residues are either amphiphilic (ile, Phe and Trp) with hydrophobic and hydrophilic domains or bi-amphiphilic (Glu, Lys and Tyr) with a hydrophobic core and two hydrophilic extremities (see article Van Eyck et al.). 


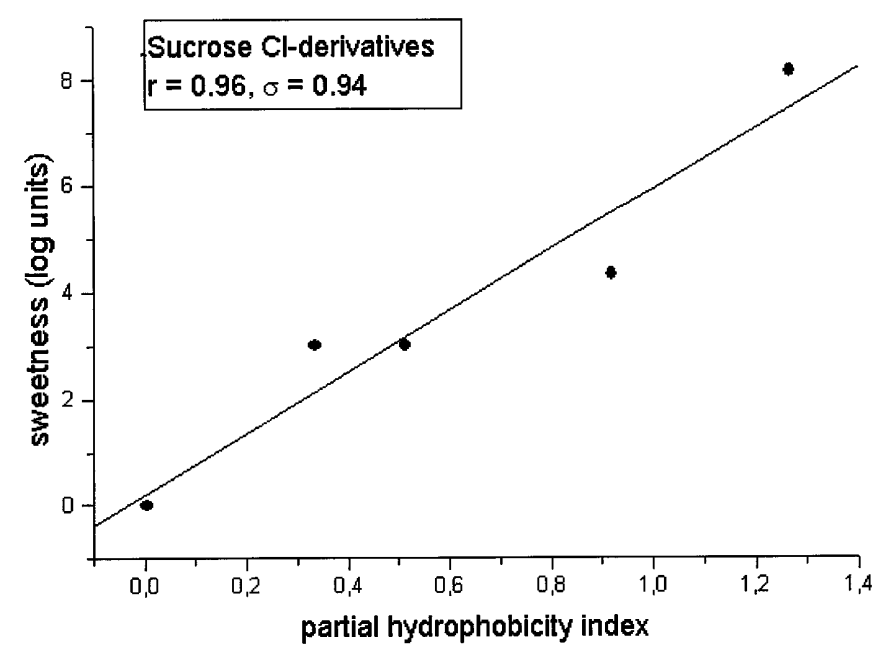

Figure 1. Sweetness of sucrose and various chlorine derivatives (as in Fig. 2) as a function of the partial hydrophobicity index (log $P$-value) which was calculated as a surface integral of the MOLFESD over the hydrophobic surface areas shown in figure 2.

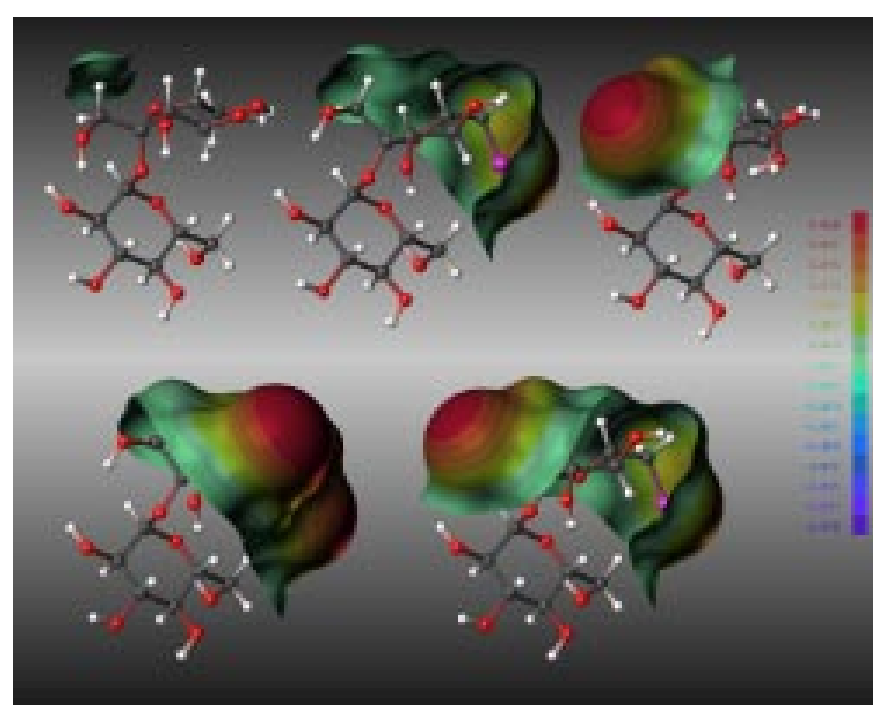

Figure 2. Hydrophobic parts of the molecular surfaces of sucrose (left, top) and various chlorine derivatives (replacements at 1 ', 4', and 6 ') with a mapping of the MOLFESD values (see article Brickmann and Jäger).

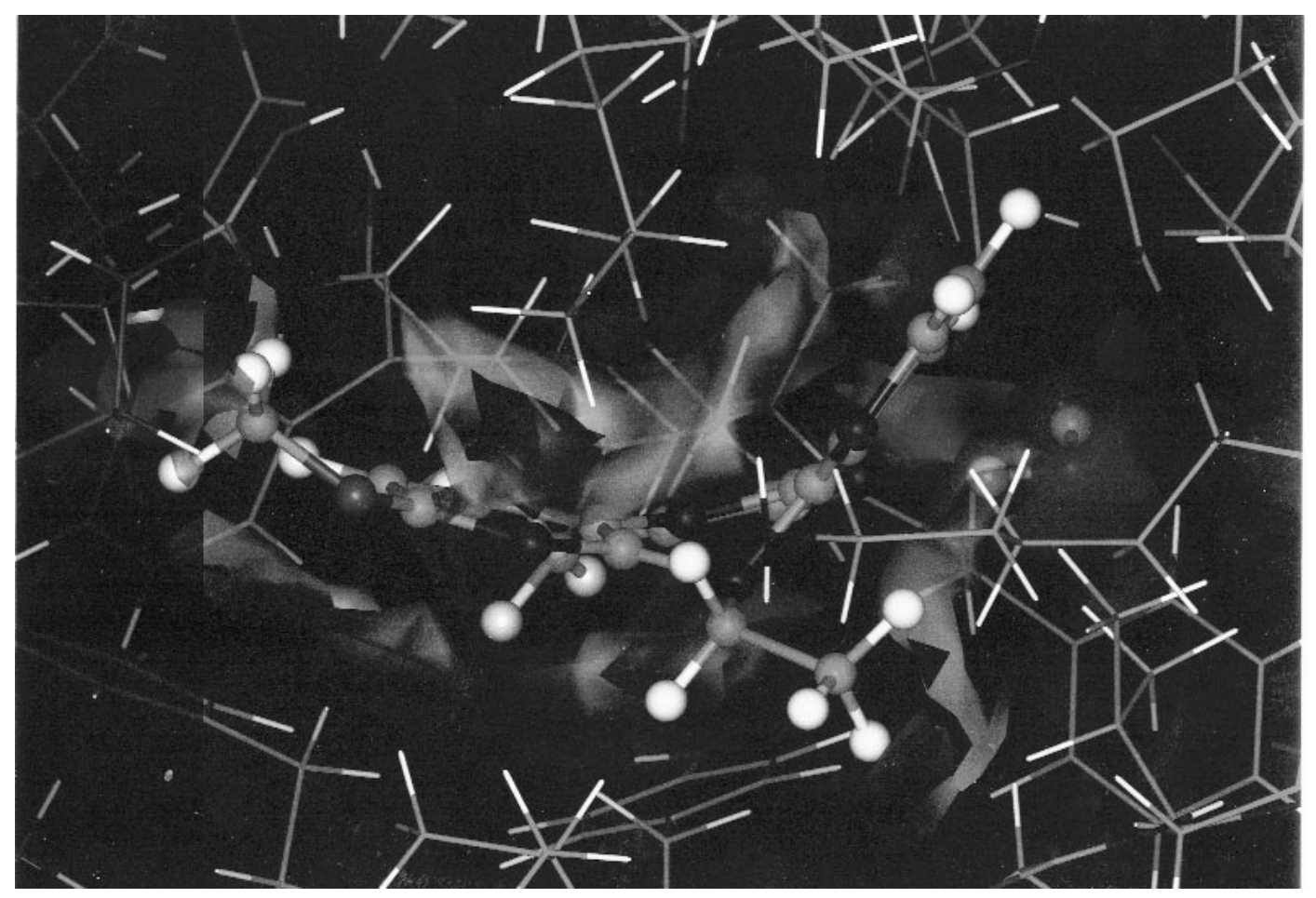

Figure 1. HINT interaction contour map for the HIV-1 Reverse Transcriptase inhibitor UK-129,485 (imidazodipyridodiazepine) bound at the RT non-nucleoside site. The contour surfaces represent interactions between the ligand and protein. Red contours indicate areas of unfavorable polar interactions (generally base-base or acid-acid); blue contours indicate areas of favorable polar interactions (generally hydrogen bonds or acid-base); purple contours indicate areas of hydrophobic-polar interaction (considered by the HINT model to be unfavorable); and green contours indicate areas of hydrophobic-hydrophobic interaction (see article Kellogg and Abraham). 

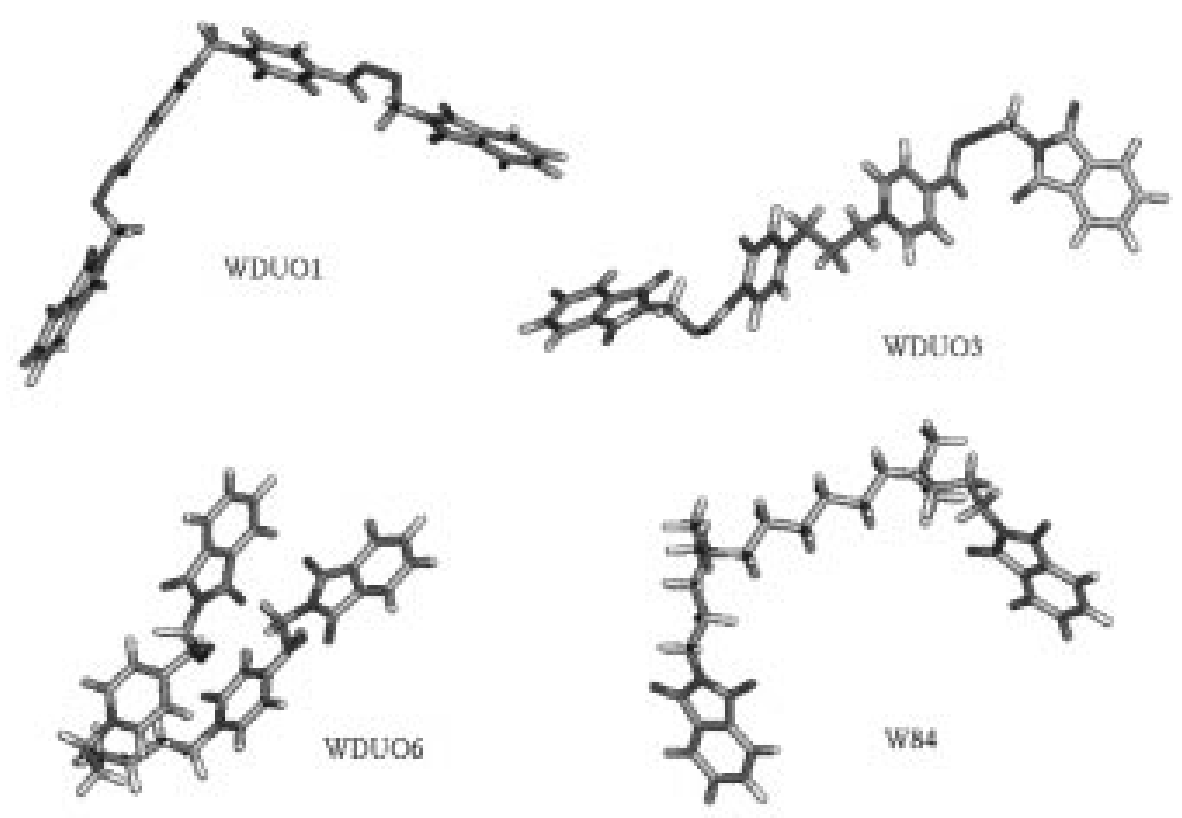

Figure 1. Best conformers of WDUO1, WDUO3, WDUO6 and W84 (see article Vistoli et al.).

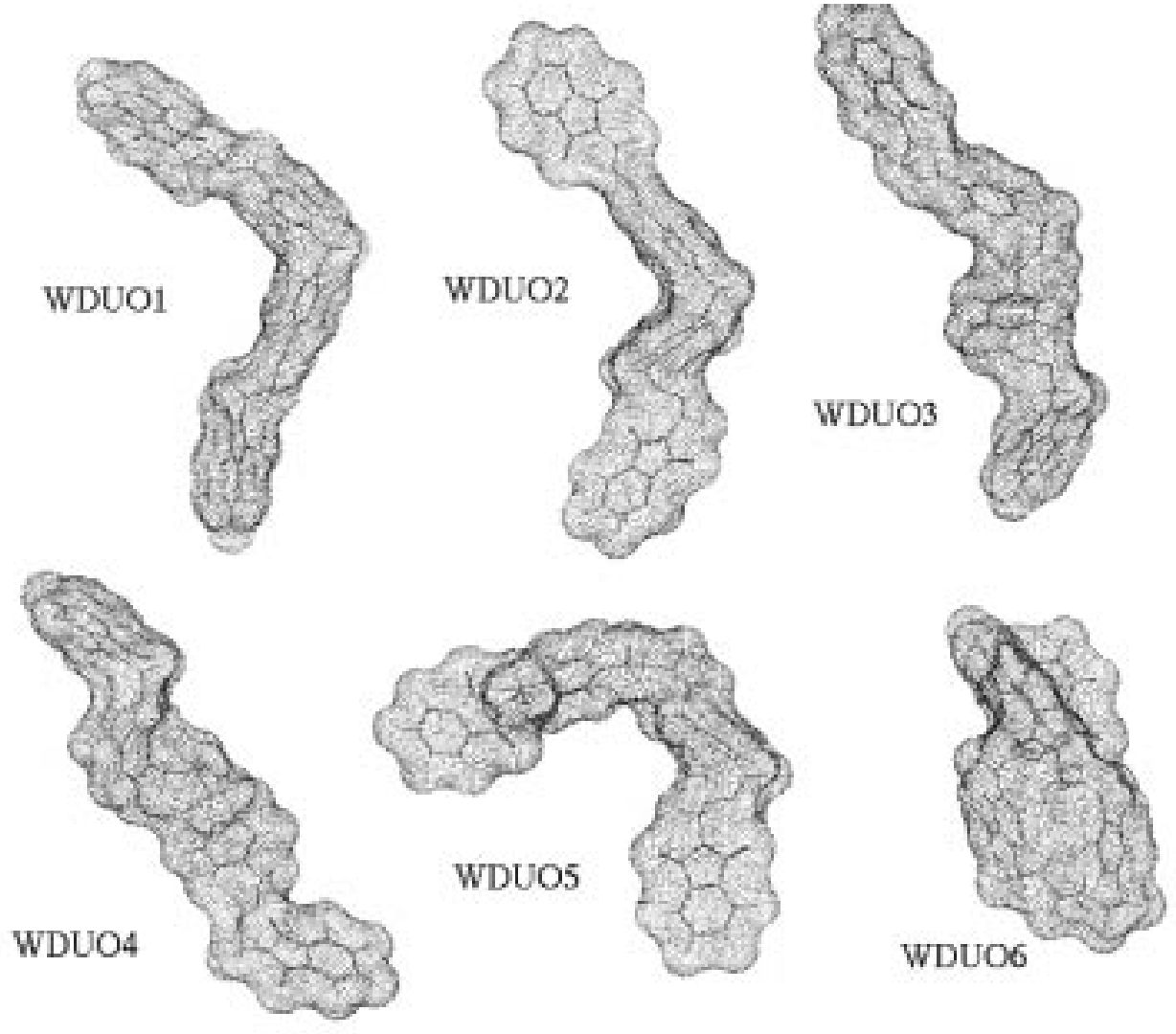

Figure 4. Hydropathicity maps (red for hydrophobic portions and blue for hydrophilic portions) of WDUO compounds (see article Vistoli et al.). 


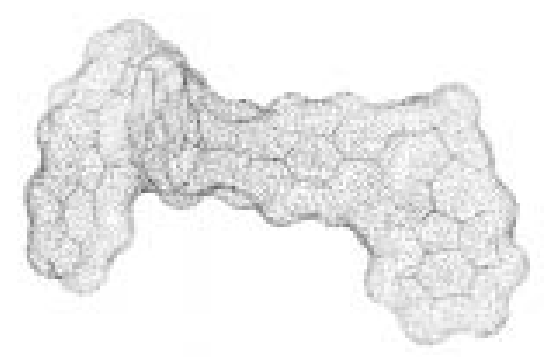

W3DUO3

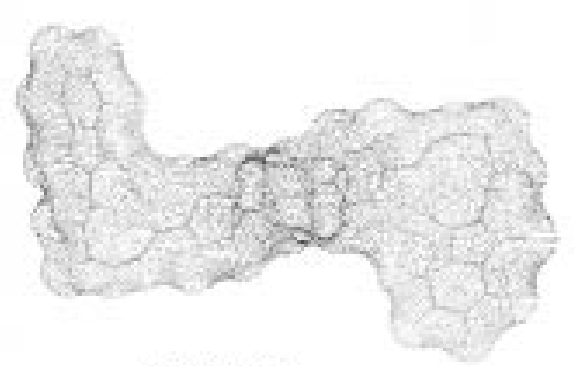

W3DUO4

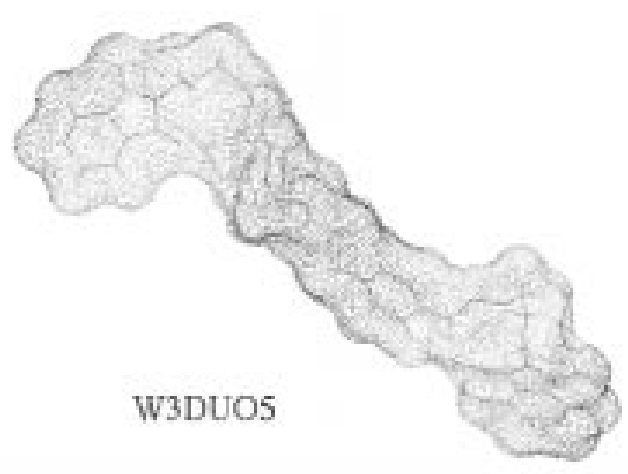

Figure 5. Hydropathicity maps of W3DUO compounds (see article Vistoli et al.).

Alcuronium:

Hydropathicity map
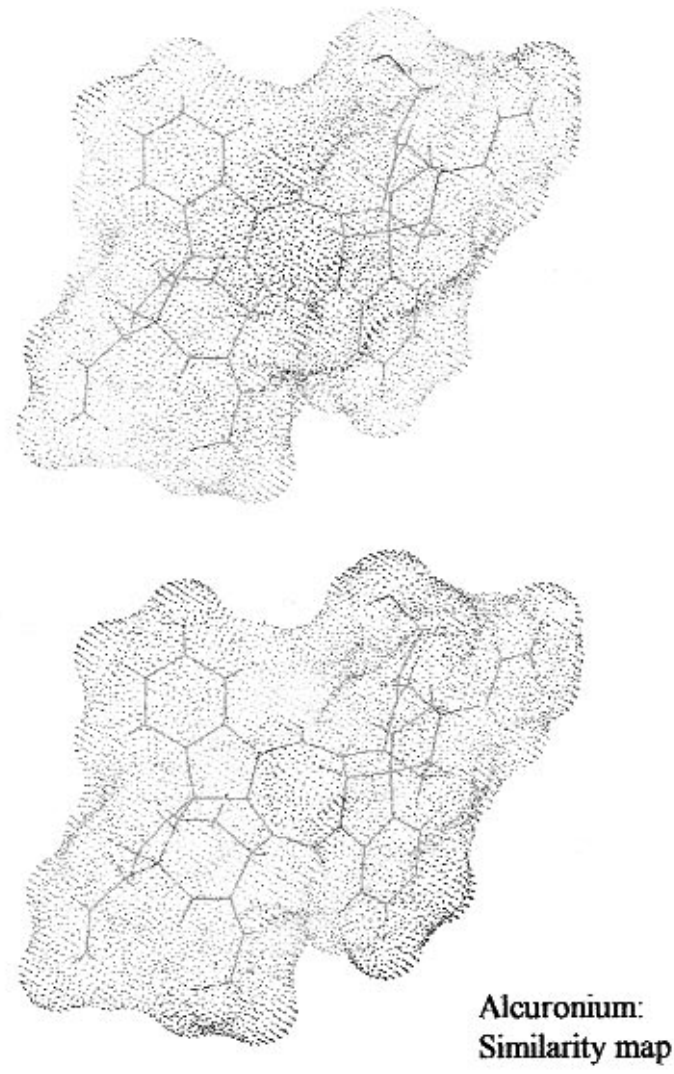

Figure 6. Hydropathicity and similarity maps of Alcuronium template (see article Vistoli et al.). 